\title{
ORGANIZAÇÃO DO CONHECIMENTO E ARQUIVOLOGIA: UMA ANÁLISE DE DOMÍNIO NOS PERIÓDICOS KNOWLEDGE ORGANIZATION E SCIRE
}

\author{
KNOWLEDGE ORGANIZATION AND ARCHIVAL \\ SCIENCE: A DOMAIN ANALYSIS IN KNOWLEDGE \\ ORGANIZATION E SCIRE JOURNALS
}

\author{
Natália Bolfarini Tognolia \\ Amanda Marissa Soares da Silvab \\ Andrieli Pachu da Silvac
}

\begin{abstract}
RESUMO
Introdução: Partindo de uma análise de domínio, o presente artigo busca iniciar a caracterização do estado da arte da Arquivologia no âmbito da Organização do Conhecimento (OC). Objetivos: Identificar as temáticas arquivísticas nas perspectivas epistemológica, aplicada e cultural em dois importantes veículos de divulgação científica: os periódicos Knowledge Organization e Scire: organización y representacíon del conocimiento. Identificar os autores que produzem nos domínios circunscritos e suas influências teóricas. Metodologia: Pesquisa exploratória e bibliográfica, de natureza quali-quantitativa. Trata-se de uma análise de domínio combinando as abordagens epistemológica, histórica e bibliométrica (análise de citação). Resultados: Demonstram um equilíbrio dos estudos epistemológicos e aplicados, e uma carência de estudos ligados aos aspectos culturais e sociais. No tocante às temáticas, observa-se a predominância de estudos que abordam as tecnologias da informação na organização e recuperação dos documentos digitais, com ênfase nos estudos sobre a classificação. A análise bibliométrica identificou Duranti e Hjørland como os autores mais influentes nas publicações, confirmando sua importância nas discussões sobre a Arquivologia e a Organização do Conhecimento. Conclusões: Identificou-se uma expressiva presença de uma comunidade discursiva ibero-americana nas discussões que buscam aproximar ambas as áreas, evidenciando o pioneirismo espanhol, português e brasileiro na inserção dos estudos arquivísticos no âmbito da organização do conhecimento.
\end{abstract}

Descritores: Organização do Conhecimento. Arquivologia. Análise de Domínio.

a Professora do Departamento de Ciência da Informação e do Programa de Pós-Graduação em Ciência da Informação da Universidade Federal Fluminense (UFF). E-mail: nataliatognoli@id.uff.br.

b Mestranda do Programa de Pós-graduação em Ciência da Informação da Universidade Federal Fluminense (UFF). E-mail: amandamarissa1@gmail.com.

c Mestre e Doutoranda em Ciência da Informação do Programa de Pós-Graduação em Ciência da Informação da Universidade Estadual Paulista Julio de Mesquita Filho (UNESP). E-mail: andripachu@gmail.com.

Inf. Inf., Londrina, v. 24, n. 3, p. 52 - 77, set./dez. 2019.

http://www.uel.br/revistas/informacao/ 
Knowledge Organization. Scire.

\section{INTRODUÇÃO}

Partindo da concepção de Smiraglia (2011) sobre a análise de domínio enquanto um estudo dos aspectos teóricos de dado entorno, representado, muitas vezes, por meio de uma literatura ou comunidade científica, busca-se, com esse estudo, iniciar a caracterização do estado da arte da Arquivologia no âmbito da Organização do Conhecimento - OC, a partir da identificação das temáticas arquivísticas nas perspectivas epistemológica, aplicada e cultural da $\mathrm{OC}$.

Para tanto, o presente trabalho verifica como se efetiva essa aproximação analisando a presença das temáticas arquivísticas no campo da Organização do Conhecimento a partir de uma análise de domínio em dois importantes veículos de divulgação científica da área: o periódico Knowledge Organization (KO), e o periódico Scire: organización y representacíon del conocimiento.

Acrescenta-se a isso uma análise bibliométrica que busca, por meio da análise de citações, identificar os marcos teóricos que fundamentam os trabalhos que confluem Arquivologia e Organização do Conhecimento, no domínio circunscrito.

A escolha dos periódicos justifica-se por tratar-se de dois veículos de divulgação científica de notória importância para a OC; a KO por ser a primeira publicação (desde 1974, ainda sob o nome de International Classification) dedicada exclusivamente à área da OC, além de estar vinculada à International Society for Knowledge Organization (ISKO) e ser indexada nas principais bases de dados. A Scire, por sua vez, merece destaque por seu protagonismo no contexto ibero-americano uma vez que seus números estão vinculados ao Ibersid (Encuentros Internacionales sobre Sistemas de Información y Documentación), evento anual da Universidade de Zaragoza que, desde 1995, tem figurado como um espaço de interlocução entre o Brasil e Espanha acerca das questões relacionadas aos sistemas de informação e documentação e, consequentemente, da OC. 
É importante destacar que a crescente presença de trabalhos publicados nos anais da ISKO Internacional e nos capítulos regionais da ISKO, como, por exemplo, o brasileiro e o ibérico, bem como no número especial recentemente publicado na Knowledge Organization, dedicado às relações entre a Arquivologia e a OC, demonstra a intenção da comunidade científica arquivística em estreitar e fortalecer as relações com a OC.

\section{ORGANIZAÇÃO DO CONHECIMENTO E ARQUIVOLOGIA: PRIMEIRAS APROXIMAÇÕES}

O conhecimento possui duas concepções: aquele passível de ser registrado (documento) e aquele que é inerente do processo individual (mente) (DAHLBERG, 1993). De acordo com Guimarães (2008) esse conhecimento é concebido a partir de um processo informativo helicoidal, segundo o qual um conhecimento que é produzido, registrado, organizado, socializado e apropriado, irá gerar um novo conhecimento que também passará pelo mesmo movimento, constituindo um fluxo helicoidal de informações infinito.

No que tange à Organização do Conhecimento, disciplina de formação recente (década de 1970), os processos de classificação, indexação e catalogação, irão incidir sobre a primeira concepção, ou seja, sobre aquele conhecimento que poder ser registrado, socializado e publicado.

De acordo com Hjørland (2008) a OC é um campo de pesquisa, ensino e prática [...] que aborda a descrição, representação e organização de documentos e de suas representações bem como de assuntos e conceitos ligados aos seres humanos e aos programas de computador. Ainda segundo o autor, dois aspectos nucleares da OC são: os processos de organização - compreendidos como a catalogação, a análise de assunto, a indexação - e os sistemas de organização - sistemas de classificação, lista de cabeçalhos de assunto, ontologias e sistemas de metadados, por exemplo, (HJØRLAND, 2008).

Guimarães (2017) a partir de estudos realizados por Arboit (2014) argumenta acerca das três dimensões de pesquisa que caracterizam a construção científica da área de OC: epistemológica, aplicada e cultural. $\mathrm{Na}$ 
primeira, encontram-se as bases conceituais, históricas e metodológicas da organização do conhecimento e seus diálogos interdisciplinares e sua produção científica; na segunda estudam-se os modelos, formatos, instrumentos, produtos e estruturas em organização do conhecimento; na terceira encontram-se os estudos ligados às questões sociais, políticas, éticas, educativas e contextuais da organização do conhecimento.

Barité (2000) considera a OC uma disciplina de convergência, uma vez que, nutrindo-se de outras áreas do conhecimento para a construção de seu arcabouço teórico-metodológico, mantém vínculos com as diversas áreas que se ocupam da produção do conhecimento científico (como Sociologia da Ciência e Filosofia da Ciência, por exemplo). Além disso, estabelece relações, também, com as áreas de Linguística, Ciência da Informação, Documentação e Inteligência Artificial.

Ainda segundo Barité (1997, p. 106), a OC estuda "as leis, os princípios e os procedimentos pelos quais se estrutura o conhecimento especializado em qualquer disciplina". Se compreendermos o conhecimento arquivístico enquanto um conhecimento especializado sobre determinado fundo (TOGNOLI et al., 2013), então podemos concluir que no âmbito dos estudos arquivísticos as funções classificação e descrição representam os processos de organização do conhecimento, enquanto que os planos de classificação e quadros de arranjo, bem como os esquemas de metadados para garantir a autenticidade de documentos arquivísticos digitais, corresponderiam aos sistemas de organização do conhecimento.

No tocante à Arquivologia, as questões que emergem após as mudanças nas formas de produzir, organizar, recuperar e acessar os documentos em meio digital, modificando as atribuições do profissional arquivista das atribuições de guardião de documentos para partícipe na gestão de documentos, na prestação de contas pelo governo e na promoção de justiça social, levaram a área um repensar necessário de sua teoria e prática, ambas atreladas às técnicas do fazer desde as publicações de seus primeiros manuais.

Embora seus princípios e conceitos ainda se mantenham válidos e devam ser respeitados - como o conceito de fundo, o princípio da proveniência e o 
conceito de documento de arquivo - a área desloca-se, agora, do paradigma custodial, indo em direção a uma abordagem que privilegia, além da custódia, o teor social implicado nos processos de produção, organização e uso de documentos de arquivo - ressaltando, aqui a importância da interlocução com outras disciplinas, como a OC.

A aproximação entre as duas áreas, notadamente por meio do conceito de conhecimento arquivístico (TOGNOLI; GUIMARÃES; TENNIS, 2013), permite que a Arquivologia se beneficie dos estudos já consolidados da área de $\mathrm{OC}$, em especial aqueles relacionados aos dilemas éticos nos processos de organização e representação do conhecimento (Fox; Reece, 2012; Milani; Pinho, 2012), à indexação (Albrechtsen, 1993; Fujita, 2003) e aos formatos e padrões interoperáveis para representação de dados (Zeng, 2019), para mencionar apenas alguns.

Em 1995, Esteban Navarro defendeu uma aproximação dos estudos arquivísticos à Organização do Conhecimento, considerando essa última um campo integrador, capaz de ir além da divisão tripartida entre as áreas de Biblioteconomia, Arquivologia e Documentação, pressupondo uma integração entre elas a partir do estudo das técnicas de armazenamento, tratamento e recuperação de documentos.

Após as considerações de Esteban Navarro, as discussões sobre a integração dos processos de $O C$ às funções arquivísticas de classificação e descrição, permaneceram adormecidas na área por vinte anos, quando Guimarães e Tognoli (2015) recuperam as discussões sobre a Arquivologia no âmbito da $\mathrm{OC}$ em seu artigo sobre o princípio da proveniência enquanto uma abordagem de análise de domínio, onde acrescentam os estudos da proveniência às onze abordagens propostas por Hjørland (2002).

Ao propor o princípio da proveniência enquanto uma abordagem de análise de domínio, Guimarães e Tognoli (2015) justificam a própria organização do conhecimento arquivístico enquanto um domínio.

É importante observar que a organização do conhecimento arquivístico tem seu núcleo na classificação (estrutural ou funcional) e nos procedimentos de descrição, com o objetivo de estabelecer prerrogativas de conhecimento. Essas prerrogativas 
são construídas de acordo com o contexto da criação dos documentos, com o objetivo de garantir seus valores probatórios e históricos. Considerando essas questões, a organização do conhecimento arquivístico torna-se um domínio composto por um conjunto de pesquisadores de diferentes partes e instituições do mundo, que integram um colégio invisível profundamente envolvido na construção de uma base epistemológica ao utilizar uma estrutura discursiva específica que funde a terminologia tradicional da arquivologia com aquela da Ciência da Informação (GUIMARÃES; TOGNOLI, 2015, p. 567).

Recentemente, Tognoli, Rodrigues e Guimarães (2019), na busca por uma definição do conhecimento arquivístico, elaboraram uma estrutura conceitual a partir da Teoria do conceito de Dahlberg (1978), tendo como base o conceito de fundo, o conhecimento da forma documental e do contexto de produção dos documentos. Segundo os autores:

[...] o conhecimento arquivístico pode ser concebido a partir do conceito de fundo (sobre o qual incidirão todos os processos de organização), caracterizado a partir da reunião e análise de documentos produzidos por uma mesma pessoa ou instituição, com base no método diplomático enquanto suporte para a metodologia da identificação arquivística (TOGNOLI, RODRIGUES E GUIMARÃES, 2019, p. 71).

Nesse contexto, além dos recentes estudos mencionados acima, destacam-se os trabalhos de Barros e Sousa (2019), Tognoli e Barros (2015), Alencar e Cervantes (2019) e Lehmkuhl et al. (2019), entre outros, emergindo enquanto uma comunidade discursiva comprometida na construção de conhecimento teórico-metodológico que auxilie a Arquivologia na produção, organização e uso dos documentos de arquivo, notadamente a partir dos estudos da OC.

\section{PROCEDIMENTOS METODOLÓGICOS}

Segundo Mai (2005, p. 605) um domínio pode ser entendido "como uma área de especialidade, um conjunto literário ou um grupo de pessoas trabalhando juntas em uma organização". Portanto, consideramos a análise de domínio enquanto um paradigma teórico e metodológico na organização do conhecimento (SMIRAGLIA, 2015) que, aqui, utilizamos para analisar os periódicos KO e Scire. 
Desta feita, o presente trabalho realiza uma análise de domínio combinando as abordagens epistemológica, histórica e bibliométrica. A abordagem epistemológica é, segundo Hjørland (2008, p. 439), "a mais básica sem a qual qualquer outra abordagem tende a ser superficial", uma vez que fornece o conhecimento sobre as bases do domínio e, geralmente, vem acompanhada da histórica. Já a abordagem bibliométrica nos permite medir a produção científica sobre Arquivologia na Organização do conhecimento, reconhecendo seus atores e influências.

Foram analisados 47 artigos publicados entre 1995 e 2019 em ambas as revistas. O recorte temporal foi feito com base na data da primeira publicação da Scire (1995), a fim de garantirmos um equilíbrio entre as publicações, uma vez que a revista KO teve seu primeiro número publicado em 1974 (ainda sob o nome International Classification).

Inicialmente foram realizadas buscas pelos termos arquivo e arquivologia/arquivística nos idiomas português e espanhol (archivo/archivistica) na Scire e em inglês na KO (archive/archival science) nos títulos e resumos dos trabalhos. Foram recuperados 28 artigos na Scire e 19 na KO.

Os trabalhos foram agrupados por revista, segundo as categorias que representam três dimensões de pesquisa que caracterizam a construção científica da área de OC: epistemológica, aplicada e cultural (relacionados aos aspectos sociais, políticos, econômicos, éticos).

Com base nos títulos, resumos e palavras-chave, identificamos as temáticas mais recorrentes e as possíveis correntes de pesquisa no âmbito da $\mathrm{OC}$, no que tange aos arquivos e à Arquivologia.

Por fim, utilizamos a análise de citação a fim de identificarmos os autores mais produtivos e os clusters.

\section{RESULTADOS E DISCUSSÃO DOS DADOS}

A categorização de todos os artigos recuperados nas três categorias de abordagens propostas demonstrou um equilibrio entre as dimensões epistemológica e aplicada, cada uma com $42,55 \%$ de trabalhos e uma pequena 
quantidade de trabalhos que abordam os aspectos culturais e sociais $(14,9 \%)$, conforme demonstrado no gráfico a seguir:

\section{Gráfico 1- Dimensões}

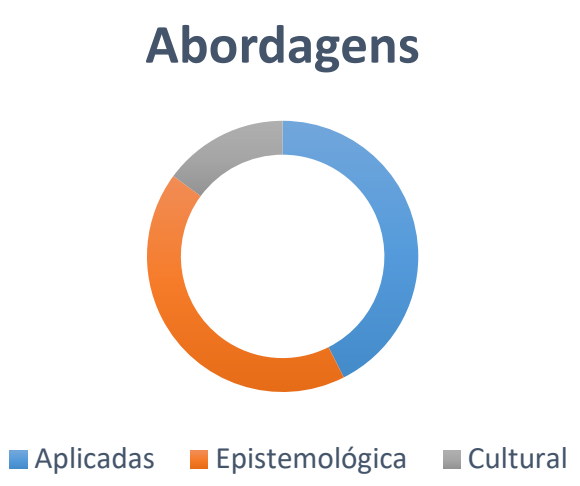

Fonte: Elaborado pelos autores.

É importante destacar que a dimensão epistemológica apresenta uma frequência maior com relação às demais na KO (8 artigos de 19, 42\%), enquanto que na Scire (46\%), observa-se uma predominância - ainda que pequena - da dimensão aplicada com 13 artigos de $28(46,43 \%)$ o que demonstra, destarte, uma preocupação dos autores em construir e promover os estudos interdisciplinares e o desenvolvimento das bases conceituais, históricas e epistemológicas da Arquivologia no âmbito dos estudos da OC, bem como metodologias para a aplicação.

$\mathrm{Na}$ dimensão epistemológica destacam-se os estudos relacionados às temáticas da classificação arquivística (Foscarini, 2006; Martínez Comeche, 1997; Henttonen, 2015; Sales, 2016; Barros e Moraes, 2010; Simões et al., 2016), dos documentos digitais (Cruz Mundet, 1997; Bustelo, 1997; Gómez, 2005; Díaz e Palacios, 2006) da descrição arquivística (Moreno et al., 2005; Díaz e González, 2005; Barros e Sousa, 2019), da Diplomática (Tognoli, 2015; Troitiño, 2011; Tognoli; Rodrigues; Guimarães, 2019) e dos princípios da Arquivologia (SILVA; RIBEIRO, 2012), com ênfase nas duas primeiras temáticas - classificação e documentos digitais -, onde se observa um maior número de publicações (com 6 e 4 artigos, respectivamente) conforme demonstram as 
tabelas a seguir:

Tabela 1 - Artigos da dimensão epistemológica

Temática classificação arquivística

\begin{tabular}{|l|l|l|}
\hline Título & Autor & Periódico \\
\hline $\begin{array}{l}\text { Clasificaciones bibliotecarias y } \\
\text { archivísticas: } \begin{array}{l}\text { diferencias y } \\
\text { semejanzas en la organización del } \\
\text { conocimiento. }\end{array}\end{array}$ & Scire \\
\hline $\begin{array}{l}\text { A classificação arquivística: aspectos } \\
\text { teóricos de sua constituição. }\end{array}$ & $\begin{array}{l}\text { BARROS; } \\
\text { MORAES }\end{array}$ & Scire \\
\hline $\begin{array}{l}\text { Clasificaciones documentales y } \\
\text { procesos informativos: una vía de } \\
\text { racionalizaciones. }\end{array}$ & COMECHE, J. & Scire \\
\hline $\begin{array}{l}\text { Records Classification and } \\
\text { Functions: An Archival Perspective }\end{array}$ & FOSCARINI, & $\mathrm{KO}$ \\
\hline $\begin{array}{l}\text { Dimensions of Contextual Records } \\
\text { Management Classifications }\end{array}$ & HENTTONEN & $\mathrm{KO}$ \\
\hline $\begin{array}{l}\text { Theory of Classification and } \\
\text { Classification in Libraries and } \\
\text { Archives: SIMÕES et al. } \\
\text { divergences convergences and }\end{array}$ & $\mathrm{KO}$ \\
\hline
\end{tabular}

Fonte: Elaborado pelos autores.

A predominância dos estudos sobre classificação na dimensão epistemológica evidencia e efetiva o aporte da OC aos estudos arquivísticos contemporâneos - haja vista que as funções classificação e descrição (temática também presente nos trabalhos) são consideradas nucleares no processo de organização do conhecimento arquivístico, conforme demonstrado anteriormente.

Tabela 2 - Artigos da dimensão epistemológica

Temática documentos digitais

\begin{tabular}{|l|l|l|}
\hline Título & Autor & Periódico \\
\hline $\begin{array}{l}\text { Los documentos electrónicos de } \\
\text { archivo: pautas para la realización del }\end{array}$ & DÍAZ; & Scire \\
Nuevo diccionario de archivística & PALACIOS & \\
\hline $\begin{array}{l}\text { Archivos y metadatos de conservación: } \\
\text { estado del arte y propuesta } \\
\text { metodológica }\end{array}$ & & \\
\hline
\end{tabular}




\begin{tabular}{|l|l|l|}
\hline $\begin{array}{l}\text { Los sistemas de gestión electrónica de } \\
\text { la documentación y la teoría del ciclo } \\
\text { vital de los documentos en las }\end{array}$ & & Scire \\
organizaciones & \\
\hline $\begin{array}{l}\text { Los archivos electrónicos en España: } \\
\text { del gran hermano al control } \\
\text { demográfico }\end{array}$ & MUNDET, J. & \\
\hline
\end{tabular}

Fonte: Elaborado pelos autores

Importante destaque se dá, também, às publicações cujas temáticas centram-se nos documentos e ambientes digitais, o que corrobora a preocupação dos autores em abordar as questões contemporâneas sobre a gestão, preservação e acesso ao conhecimento arquivístico em ambiente digital, indo ao encontro dos estudos atuais no âmbito da OC (Zeng, 2019; Finnemann, 2019) que, com a era digital, assistiu à emergência de normas, padrões e metadados específicos para lidar com os documentos em ambientes digitais, interativos e dinâmicos.

$\mathrm{Na}$ dimensão aplicada, predominam os trabalhos sobre os Sistemas de Organização do Conhecimento (SOC) definidos por Mazzocchi (2018) como uma ampla gama de itens, caracterizados por diferentes tipos de estrutura e função, variáveis com relação às tecnologias e utilizados em diferentes contextos e comunidades, "cujo objetivo é apoiar a organização do conhecimento e da informação para assegurar seu gerenciamento e recuperação" (MAZZOCCHI, 2018, p. 54).

No âmbito dos estudos arquivísticos, são considerados SOC os planos de classificação e quadros de arranjos, as normas de descrição arquivística, os instrumentos de pesquisa - tais como inventários e catálogos - e padrões de metadados.

Nesse contexto, as temáticas predominantes nos SOC presentes na dimensão aplicada são a descrição arquivística - como os estudos sobre a aplicação de normas e padrões de descrição (Bonal Zazo, 2000; Brun, 2011; Prado Martinéz, 2019) e criação de instrumentos de pesquisa e padrões para a recuperação e acesso de documentos em ambiente digital (Lopes Yepes, 2008; Chen, 2019) - e a classificação - com trabalhos que propõem a elaboração de quadros de classificação (Silva; Schmidt, 2018; Morales et al., 2015; Walt, 2004; 
Ribeiro, 2014), ou interoperabilidade entre sistemas (Marcondes, 2019) conforme demonstram as tabelas a seguir:

Tabela 3 - Artigos da dimensão aplicada

\section{Temática descrição arquivística}

\begin{tabular}{|c|c|c|}
\hline Título & Autor & Periódico \\
\hline $\begin{array}{l}\text { Catálogo Colectivo de las Colecciones de } \\
\text { Mapas, Planos y Dibujos de los Archivos } \\
\text { Estatales: Nuevos proyectos en el Archivo } \\
\text { General de Simancas }\end{array}$ & GUERRA, J. & Scire \\
\hline $\begin{array}{l}\text { Aplicabilidad de EAD en archivos históricos } \\
\text { de ingeniería: el fondo Carlos Fernández } \\
\text { Casado }\end{array}$ & BRUN, R. & Scire \\
\hline $\begin{array}{l}\text { Filmotecas y archivos fílmicos en línea: } \\
\text { producción, difusión, interconexión y } \\
\text { posicionamiento en Internet }\end{array}$ & $\begin{array}{l}\text { LOPES YEPES, } \\
\text { A. }\end{array}$ & Scire \\
\hline $\begin{array}{l}\text { El archivo municipal de San Javier : } \\
\text { propuesta de descripción del fondo } \\
\text { fotográfico }\end{array}$ & $\begin{array}{l}\text { BAIXAULI, L. et } \\
\text { al. }\end{array}$ & Scire \\
\hline $\begin{array}{l}\text { Sistema de información histórica para la } \\
\text { colección de papeles varios del Archivo } \\
\text { Histórico de la Universidad de Salamanca }\end{array}$ & MORENO, A. V. & Scire \\
\hline $\begin{array}{l}\text { Correspondencias entre la norma aragonesa } \\
\text { para la descripción de autoridades de } \\
\text { archivos (ARANOR) y MARC21: hacia la web } \\
\text { semántica }\end{array}$ & $\begin{array}{l}\text { PRADO } \\
\text { MARTINÉZ, M. } \\
\text { A. }\end{array}$ & Scire \\
\hline $\begin{array}{l}\text { Padronização: base da análise documental } \\
\text { nos arquivos }\end{array}$ & BONAL ZAZO, J. & Scire \\
\hline $\begin{array}{l}\text { Designing a Thesaurus to Give Visibility to the } \\
\text { Historical Archives in the Archivo del Reino in } \\
\text { Valencia }\end{array}$ & $\begin{array}{l}\text { GIMÉNEZ- } \\
\text { CHORNET, V.; } \\
\text { ESCRIG- } \\
\text { GIMÉNEZ, M. }\end{array}$ & $\mathrm{KO}$ \\
\hline $\begin{array}{l}\text { Childhood and Adolescence Between Past } \\
\text { and Present. Using Knowledge Organization } \\
\text { to Bridge the Different Channels of a Cultural } \\
\text { Institution: The Case of the Istituto degli } \\
\text { Innocenti, Firenze }\end{array}$ & ROSATI, L. et al. & $\mathrm{KO}$ \\
\hline $\begin{array}{l}\text { The Representation of Archival Information in } \\
\text { Controlled Vocabularies: The Context of the } \\
\text { Archival Institutions in Rio de Janeiro }\end{array}$ & SOUZA, R. M. & $\mathrm{KO}$ \\
\hline
\end{tabular}

Fonte: Elaborado pelos autores 
Tabela 4 - Artigos da dimensão aplicada

Temática classificação

\begin{tabular}{|l|l|l|}
\hline Título & Autor & Periódico \\
\hline $\begin{array}{l}\text { El proceso de elaboración del cuadro de } \\
\text { clasificación de los archivos de Machado } \\
\text { de Assis y Rui Barbosa: análisis crítico y } \\
\text { comparativo }\end{array}$ & $\begin{array}{l}\text { SILVA, L.; } \\
\text { SCHMIDT, C. }\end{array}$ & Scire \\
\hline $\begin{array}{l}\text { Archivo de Espacio P: Propuesta de un } \\
\text { cuadro de clasificación para su Continuidad } \\
\text { Digital / Espacio P Archive: Classification } \\
\text { Table proposal for Digital Continuity }\end{array}$ & MORALES, F. et & Scire \\
\hline $\begin{array}{l}\text { Uma Classificação de tipos de objetos de } \\
\text { patrimônio para integração de acervos } \\
\text { digitais de arquivos, bibliotecas e museus }\end{array}$ & MARCONDES, & Scire \\
\hline $\begin{array}{l}\text { A Classification Scheme for the } \\
\text { Organization of Electronic Documents in } \\
\begin{array}{l}\text { Small, Medium and Micro Enterprises } \\
\text { (SMMEs) }\end{array}\end{array}$ & WALT, M. & KO \\
\hline $\begin{array}{l}\text { The Use of Classification in Archives as a } \\
\text { Means of Organization, Representation and } \\
\text { Retrieval of Information }\end{array}$ & RIBEIRO, F. & KO \\
\hline
\end{tabular}

Fonte: Elaborado pelos autores

Nota-se que, em alguns casos, o trabalho apresenta mais de uma temática, confluindo a descrição arquivística e a classificação aos documentos digitais, por exemplo, o que demonstra que os estudos sobre a gestão e preservação dos documentos digitais perpassam as demais temáticas ligadas aos sistemas de organização do conhecimento.

A dimensão cultural apresentou a menor incidência de trabalhos, com apenas $14,9 \%$ do total de 47 artigos recuperados. Dentre as temáticas mais presentes destaca-se a ética ligada à prática profissional (Rego et al., 2014; Silva et al, 2015) e à descrição arquivística (Gilliland, 2012; Zhang, 2012).

Tabela 5 - Artigos da dimensão cultural

\begin{tabular}{|l|l|l|}
\hline \multicolumn{1}{|c|}{ Título } & Autor & Periódico \\
\hline $\begin{array}{l}\text { Aspectos éticos de la organización del } \\
\text { conocimiento en la práctica profesional } \\
\text { archivística: un estudio de los principios éticos de } \\
\text { AAB, CIA y SAA }\end{array}$ & REGO, L. et & Scire \\
\hline $\begin{array}{l}\text { La Archivística en el ámbito de las organizaciones } \\
\text { empresariales: estado de la cuestión y }\end{array}$ & & \\
propuestas de actuación & PRADO & Scire \\
\hline
\end{tabular}




\begin{tabular}{|l|l|l|}
\hline $\begin{array}{l}\text { Estudios de usuarios en archivos municipales: } \\
\text { una aproximación teórico-práctica }\end{array}$ & $\begin{array}{l}\text { RUBIO } \\
\text { HERNÁNDE } \\
\text { Z, A. }\end{array}$ & Scire \\
\hline $\begin{array}{l}\text { Archival Context, Digital Content, and the Ethics } \\
\text { of Digital Archival Representation }\end{array}$ & ZHANG, J. & $\mathrm{KO}$ \\
\hline $\begin{array}{l}\text { Contemplating Co-creator Rights in Archival } \\
\text { Description }\end{array}$ & $\begin{array}{l}\text { GILLILAND, } \\
\text { A. }\end{array}$ & $\mathrm{KO}$ \\
\hline $\begin{array}{l}\text { Ethical Issues of Knowledge Organization in } \\
\text { Designing a Metadata Schema for the Leo Kottke } \\
\text { Archives }\end{array}$ & $\begin{array}{l}\text { MCQUEEN, } \\
\text { K. }\end{array}$ & $\mathrm{KO}$ \\
\hline $\begin{array}{l}\text { Ethical Values in Archival Arrangement and } \\
\text { Description: An Analysis of Professional Codes of } \\
\text { Ethics }\end{array}$ & $\begin{array}{l}\text { SILVA, A. et } \\
\text { al. }\end{array}$ & $\mathrm{KO}$ \\
\hline
\end{tabular}

Fonte: Elaborado pelos autores

A baixa incidência de trabalhos nessa dimensão evidencia uma carência dos estudos arquivísticos que abordem as questões culturais e sociais no âmbito da Organização do Conhecimento.

A fim de ilustrar a confluência de temas abordados nos artigos, com vistas a identificar possíveis perspectivas de pesquisa nos domínios estudados, apresenta-se, a seguir, a análise de cada domínio.

\subsection{ANÁlise de DOMÍNIO: SCIRE}

A Scire é uma revista que promulga em seu bojo os estudos da organização do conhecimento e da informação. Esta é advinda do congresso anual do Ibersid que tem como foco os estudos sobre os sistemas de informação e documentação, abarcando, a partir disso, as pesquisas relacionadas às instituições como bibliotecas, arquivos, centros de documentação entre outros.

A busca por artigos na Scire recuperou 28 documentos, sendo 12 artigos na dimensão epistemológica, 13 na aplicada e 3 na cultural. A tabela 6 demonstra as temáticas arquivísticas identificadas nos artigos:

Tabela 6 - Temáticas arquivísticas na Scire

\begin{tabular}{|c|l|}
\hline Dimensão & \multicolumn{1}{|c|}{ Temáticas mais recorrentes } \\
\hline Epistemológica & $\begin{array}{l}\text { Documento digital (4) } \\
\text { Processos de Organização do Conhecimento (5) } \\
\end{array}$ \\
Diplomática (2) \\
\hline Aplicada & $\begin{array}{l}\text { Ambiente digital de gestão e preservação de } \\
\text { documentos arquivísticos (7) } \\
\text { Processos de Organização do Conhecimento (5) }\end{array}$ \\
\hline
\end{tabular}




\begin{tabular}{|c|l|}
\hline & Sistema de Organização do Conhecimento (2) \\
& Análise diplomática (1) \\
& Documentos audiovisuais (2) \\
\hline Cultural & Ética (1) \\
& Estudos de usuário (1) \\
& Arquivos privados (1) \\
\hline
\end{tabular}

Fonte: Elaborado pelos autores

Mais uma vez, destaca-se a predominância nas dimensões epistemológica e aplicada de temáticas relacionadas aos documentos e aos ambientes digitais, evidenciando uma preocupação dos autores no domínio da Scire de abordar questões contemporâneas e emergentes no contexto da organização do conhecimento arquivístico em ambientes digitais. As temáticas da classificação e da descrição (processos de organização do conhecimento) também se apresentam como as mais recorrentes nesse domínio, muitas vezes combinadas com as temáticas digitais.

Outro fator importante de análise diz respeito à nacionalidade dos autores. Por tratar-se de um periódico espanhol ligado a um evento já consolidado na tradição ibero-americana (Ibersid), é natural que dentre os trabalhos recuperados a maioria seja de autoria espanhola (18 trabalhos) ou de autores de língua espanhola (01 da Colômbia). O segundo país com maior número de trabalhos (09) é o Brasil, com a maioria de pesquisadores da Universidade Estadual Paulista - UNESP ( 6 trabalhos) e com 03 trabalhos da Universidade Federal Fluminense - UFF.

Isso demonstra que, nos últimos anos, a revista Scire tem servido como um importante espaço de divulgação das pesquisas brasileiras que abordam as relações entre Arquivologia e a Organização do Conhecimento e que o Brasil possui uma comunidade discursiva presente na área, notadamente a partir de pesquisas realizadas na UNESP.

\subsection{ANÁlise de Domínio: KNowledge Organization}

A revista é fruto da International Society for Knowledge Organization ISKO, nascida como Society for Classification (1974). Desde então, a KO publica anualmente de 4 a 8 números, tendo sua primeira edição em 1974, quando ainda 
ligada à Society for Classification.

A busca por artigos na Knowledge Organization recuperou 19 documentos, sendo 8 artigos na dimensão epistemológica, 7 na aplicada e 4 na cultural. A tabela 7 demonstra as temáticas arquivísticas identificadas nos artigos:

Tabela 7 - Temáticas arquivísticas na KO

\begin{tabular}{|c|l|}
\hline Dimensão & \multicolumn{1}{|c|}{ Temáticas mais recorrentes } \\
\hline Epistemológica & Processos de Organização do Conhecimento \\
& $(4)$ \\
& Revisão conceitual (5) \\
& Sistemas de Organização do Conhecimento (1) \\
\hline Aplicada & Ambiente digital de gestão e preservação de \\
& documentos arquivísticos (1) \\
& Representação da informação (7) \\
& Sistemas de Organização do Conhecimento (5) \\
\hline Cultural & Ética profissional (4) \\
& Representação em ambiente digital (2) \\
& Descrição arquivística (2) \\
& Arquivos de minorias (2) \\
\hline
\end{tabular}

Fonte: Elaborado pelos autores

A dimensão epistemológica corresponde a $42 \%$ dos trabalhos publicados na KO, com a temática da classificação arquivística sendo a mais recorrente nos estudos. Na dimensão aplicada as temáticas ligadas aos documentos digitais e aos ambientes de gestão e preservação e à representação da informação são as mais recorrentes. Nessa última, merece destaque os trabalhos que abordam os tesauros como um sistema de representação nos arquivos históricos (Giménez Chornet; Escrig-Giménez, 2011; Rosati et al., 2013).

$\mathrm{Na}$ dimensão cultural, a ética é a temática mais recorrente sendo abordada junto aos códigos profissionais do arquivista (Silva et al., 2015), à ética na representação de comunidades marginalizadas (Gilliland, 2012; Zhang, 2012) e aos desafios colocados pela representação da informação em ambientes digitais (Zhang, 2012; McQueen, 2015). Vale destacar que há um número maior de temáticas do que de artigos, haja vista que os artigos abordam temas complementares.

No tocante à nacionalidade, destaca-se a forte presença de autores portugueses, espanhóis e brasileiros, confirmando a influência da comunidade 
ibero-americana na produção de conhecimento sobre as temáticas arquivísticas no âmbito da Organização do Conhecimento. Neste contexto, de 19 artigos recuperados, 42,11\% foram escritos por 13 autores ibero-americanos. Em seguida estão os autores norte-americanos que representam $26,32 \%$ da produção.

\subsection{ANÁLISE BiBLIOMÉTRICA}

Buscando identificar os autores mais citados e suas influências nos trabalhos que abordam as temáticas arquivísticas nos domínios definidos, utilizamos as 750 referências de todos os trabalhos recuperados na KO e na Scire, que excluindo as autocitações, chegou-se a um corpus de 581 autores citados. Assim, aplicando a lei de elitismo de Price, (ALVARADO, 2009) que corresponde à raiz quadrada do total de autores citados, $\sqrt{581}$, chegou-se aos 25 autores mais citados, explicitados na tabela 8:

Tabela 8 - Autores mais citados

\begin{tabular}{|c|c|}
\hline Autor & No. de citações \\
\hline Duranti, L. & 24 \\
\hline Hjørland, B. & 19 \\
\hline Bearman, D. & 12 \\
\hline Bellotto, H.L & 10 \\
\hline Couture, C. & 9 \\
\hline Guimarães, J. A. C. & 9 \\
\hline Schellenberg, T. R. & 9 \\
\hline MacNeil, H. & 8 \\
\hline Cook, T. & 8 \\
\hline Foscarini, F. & 7 \\
\hline Heredia Hererra, A. & 7 \\
\hline Guercio M. & 6 \\
\hline Rousseau, J. & 6 \\
\hline Hurley, C. & 6 \\
\hline Henttonen, P. & 5 \\
\hline McKemmish, S. & 5 \\
\hline Smiraglia, Richard P. & 5 \\
\hline Sousa, R. T. B. & 5 \\
\hline Yeo, G. & 5 \\
\hline Cook, M. & 4 \\
\hline Cook, T. & 4 \\
\hline Dahlberg, I. & 4 \\
\hline Gilliland, Anne J. & 4 \\
\hline
\end{tabular}

Fonte: Elaborado pelos autores 
No entanto, para fins de construção de redes e análise optamos por considerar apenas os autores que receberam mais de 8 citações, o que reduziu o número para 9 autores.

Figura 1 - Rede de autores mais citados

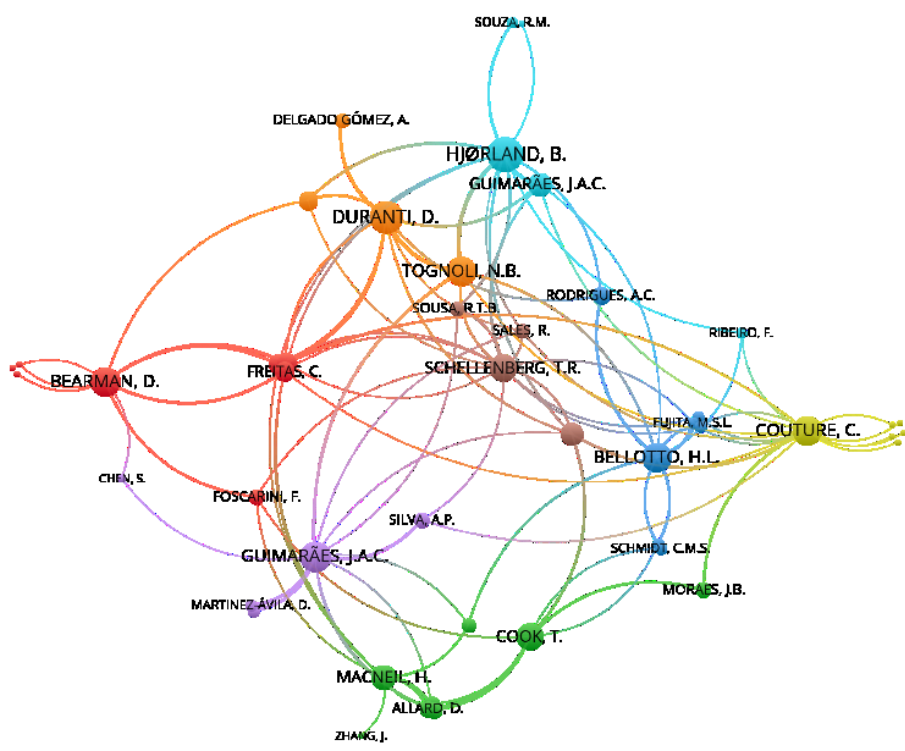

Fonte: Elaborado pelos autores com o software VOSviewer

Destaca-se, destarte, que entre os 25 autores, os mais citados são Duranti e Hjørland, o que corrobora a contribuição dos dois enquanto marcos teóricos importantes para os estudos da Arquivologia contemporânea e da Organização do Conhecimento, respectivamente. Nota-se, na rede, a proximidade desses autores aos ibero-americanos (Guimarães; Souza; Troitiño Rodriguez; Tognoli; Delgado Gomez), o que denota uma influência direta de suas obras na construção do pensamento científico nacional.

Bearman, o terceiro autor mais citado, também influencia o pensamento ibero-americano, notadamente o espanhol e português (Simões; Freitas; Rodríguez-Bravo, 2016; Bonal Zazo, 2000), sem ter recebido, no entanto, qualquer citação dos autores brasileiros.

Dentre os 25 autores mais citados, dois são brasileiros (Bellotto e Guimarães), recebendo, respectivamente, 10 e 9 citações cada. Por se tratar de 
trabalhos cujos autores ibero-americanos, como vimos nas análises anteriores, figuram entre os mais produtivos, apresentando-se como autores em 37 publicações (do total de 47 analisadas), é natural que se observe a presença de autores brasileiros citados nessas obras. No entanto, merece destaque a presença da obra de Guimarães em dois trabalhos que extrapolam o espaço ibero-americano, especificamente nos textos de Bak, Allard e Ferris (2019), e de Chen (2019), o que demonstra uma influência também em obras norteamericanas.

Outro dado interessante é o fato de Carol Couture, canadense e o quarto autor mais citado, não ter recebido nenhuma citação em obras em inglês, evidenciando que sua influência - ao menos no domínio circunscrito nesta pesquisa - limita-se aos autores ibero-americanos, o que não acontece com Terry Cook e Heather MacNeil, que influenciam tanto o pensamento iberoamericano (Silva e Schmidt, 2018; Barros e Moraes, 2010; Troitiño Rodriguez, 2015; Simões, Freitas e Rodríguez-Bravo, 2016), quanto norte-americano e europeu (Foscarini, 2006; Zhang, 2012; Bak, Allard e Ferris, 2019).

\section{CONSIDERAÇÕES}

O presente trabalho buscou verificar a presença das temáticas arquivísticas no âmbito da Organização do Conhecimento a fim de analisar como se efetiva a aproximação desta com a Arquivologia. Para tanto se recorreu a uma análise de domínio em dois veículos de divulgação científica de impacto na área: a Scire e a Knowledge Organization.

A partir de uma categorização com base nas dimensões epistemológica, aplicada e cultural, identificou-se um equilíbrio entre os estudos epistemológicos e aplicados, e uma carência de estudos ligados aos aspectos culturais e sociais, o que pode evidenciar uma frente de pesquisa inexplorada para a Arquivologia no âmbito da Organização do Conhecimento.

No tocante às temáticas, observou-se uma predominância de estudos que abordam as tecnologias da informação na organização e recuperação dos 
documentos digitais, o que demonstra uma atualização e uma preocupação dos teóricos da área com as questões contemporâneas.

Ainda no que tange às temáticas mais recorrentes, a presença da classificação arquivística e dos planos de classificação evidencia as relações existentes entre a Arquivologia e a Organização do Conhecimento, o que pode configurar um ponto de partida para o reconhecimento de um aporte teórico e metodológico da OC aos estudos arquivísticos contemporâneos, em especial àqueles ligados aos sistemas de organização do conhecimento aplicados aos documentos digitais e aos ambientes de gestão e preservação.

A análise bibliométrica constatou a presença de marcos teóricos consolidados nas duas áreas, Arquivologia e Organização do conhecimento, como Duranti e Hjørland, o que corrobora a importância desses autores para a construção de conhecimento acerca das relações entre as áreas e, também, acerca dos processos de organização e representação do que Tognoli, Rodrigues e Guimarães (2019) definem como conhecimento arquivístico.

Por fim, confirma-se a presença de uma comunidade discursiva iberoamericana nas discussões que buscam aproximar ambas as áreas, evidenciando o pioneirismo espanhol, português e brasileiro na inserção dos estudos arquivísticos no âmbito da Organização do Conhecimento.

\section{REFERÊNCIAS}

ALBRECHTSEN, H. Subject analysis and indexing: from automated indexing to domain analysis. The Indexer, London, v.18, n. 4, p. 219-24, 1993.

ALENCAR, M. F; CERVANTES, B. M. N. A semântica na organização do conhecimento arquivístico: o caso dos tesauros funcionais. In: BARROS, T. H. B; TOGNOLI, N. B. (org). Organização do Conhecimento responsável: promovendo sociedades democráticas e inclusivas. Estudos avançados em Organização do Conhecimento, v. 5. Belém: Ed. UFPA, p. 287-297, 2019.

ALVARADO, R. U. Elitismo na literatura sobre a produtividade dos autores. $\mathbf{C i}$. Inf. [online]. v. 38, n. 2, p.69-79, 2009.

ARBOIT, A. E. O processo de institucionalização sociocognitiva do domínio de organização do conhecimento a partir dos trabalhos científicos dos 
congressos da ISKO. 2014. 285 f. Tese (doutorado) - Universidade Estadual Paulista Julio de Mesquita Filho, Faculdade de Filosofia e Ciências, 2014.

BAIXAULI, L. L.; ROS, C. M. B.; HERNÁNDEZ, M. M. El archivo municipal de San Javier: propuesta de descripción del fondo fotográfico. Scire: representación y organización del conocimiento, v. 8, n. 2, p. 201-210, 2002.

BAK, G.; ALLARD, D.; FERRIS, S. Knowledge Organization as Knowledge Creation: Surfacing Community Participation in Archival Arrangement and Description. Knowledge Organization, v. 46, n. 7, 2019.

BARITÉ, M. Los conceptos y su representación: una perspectiva terminológica para el tratamiento temático de la información. Scire, v. 6, n. 1, p. 31-53, 2000.

BARITÉ, M. Referenciales teóricos vigentes en el área de tratamiento temático de la información y su expresión metodológica. In: II ENCONTRO DE DIREGENTES DOS CURSOS SUPERIORES DE BIBLIOTECONOMIA DOS PAÍSES DO MERCOSUL, 27-29 nov. 1997, Buenos Aires. Relatório técnico... Porto Alegre: ABEBD, 1998.

BARROS, T. H. B.; SOUSA, R. T. B. Archival Science and Knowledge Organization: Mapping Methodological Relationships. Knowledge Organization, v. 7, n. 46, p. 493-502, 2019.

BARROS, T. H. Bragato; MORAES, J. B. E. La clasificación archivística: aspectos teóricos e su constitución. Scire: Representación y organización del conocimiento, v. 16, n. 2, p. 31-37, 2010.

BONAL ZAZO, José Luis. La normalización: base del análisis documental en los archivos. Scire: representación y organización del conocimiento, v. 6, n. 1, p. 5575, 2000.

BRUN, R. E. Aplicabilidad de EAD en archivos históricos de ingeniería: el fondo Carlos Fernández Casado. Scire, v. 17, n. 1, 2011.

BUSTELO, Carlota. Los sistemas de gestión electrónica de la documentación y la teoría del ciclo vital de los documentos en las organizaciones. Scire: representación y organización del conocimiento, v. 3, n. 2, p. 45-53, 1997.

CHEN, S. Semantic Enrichment of Linked Archival Materials. Knowledge Organization, v. 7, n. 46, p. 530-547. 2019.

DAHLBERG, I. Knowledge organization: its scope and possibilities. Knowledge Organization, v. 20, n. 4, p. 211-222, 1993.

DAHLBERG, I. Teoria do conceito. Ci. Inf., Rio de Janeiro, vol. 7, n. 2, p. 101107, 1978. 
DÍAZ, R. G.; GONZÁLEZ, R. B. Normalización y requisitos funcionales de la descripción archivística: una propuesta metodológica. Scire: representación y organización del conocimiento, v. 11, n. 1, p. 103-112, 2005.

ESTEBAN NAVARRO, M. A. La representación y la organización del conocimiento en los archivos. In: GARCIA MARCO, F. J. (coord). Organización del conocimiento en sistemas de información y documentación: actas del I Encuentro de ISKO-España, Madrid, 4 y 5 de noviembre de 1993. 1995, p. 6590.

FINNEMANN, Niels Ole. Web archive. Knowledge Organization, v. 49, n. 1, p. 47-70, 2019.

FOSCARINI, Fiorella. Records classification and functions: an archival perspective. Knowledge Organization, v. 33, n. 4, p. 188-198, 2006.

FOX, M. J; REECE, A. Which Ethics? Whose Morality?: An Analysis of Ethical Standards for Information Organization. Knowledge Organization, v. 39, n.5, 377-383, 2012.

FUJITA, M. S. L. A identificação de conceitos no processo de análise de assunto para indexação. Revista Digital de Biblioteconomia e Ciência da Informação, v. 1, n. 1, p.32-43, 2003.

GILLILAND, Anne J. Contemplating co-creator rights in archival description. KO KNOWLEDGE ORGANIZATION, v. 39, n. 5, p. 340-346, 2012.

GIMÉNEZ-CHORNET, V.; ESCRIG-GIMÉNEZ, M.. Designing a thesaurus to give visibility to the historical archives in the Archivo del Reino in Valencia. KO KNOWLEDGE ORGANIZATION, v. 38, n. 2, p. 154-166, 2011.

GÓMEZ, A. D. Archivos y metadatos de conservación: estado del arte y propuesta metodológica. Scire: representación y organización del conocimiento, v. 11, n. 1, p. 83-101, 2005.

GÓMEZ-DÍAZ, R.; PALACIOS, G., Joaquín. Los documentos electrónicos de archivo: pautas para la realización del Nuevo diccionario de archivística. Scire, v. 12, n. 2 (jul, p. 151-164), 2006.

GUERRA, Jaime Sainz. Catálogo Colectivo de las Colecciones de Mapas, Planos y Dibujos de los Archivos Estatales: nuevos proyectos en el Archivo General de Simancas. Scire, v. 19, n. 1, 2013.

GUIMARÃES, J. A. C. A dimensão teórica do tratamento temático da informação e suas interlocuções com o universo científico da International Society for Knowledge Organization (ISKO). Revista Ibero-americana de Ciência da Informação, v. 1, n. 1, p. 77-99, 2008. 
GUIMARÃES, J. A. C. Organização do Conhecimento: passado, presente e futuro sob a perspectiva da ISKO. Informação \& Informação, Londrina, v. 22, n. 2, p. $84-98,2017$.

GUIMARÃES, J. A. C.; TOGNOLI, N. B. Provenance as a Domain Analysis Approach in Archival Knowledge Organization. Knowledge Organization, v. 42, n. 8, p. 562- 69, 2015.

HENTONNEN, Pekka. Dimensions of Contextual Records Management Classifications. KO KNOWLEDGE ORGANIZATION, v. 42, n. 7, p. 477-485, 2015.

HJØRLAND, B. Domain analysis in information science: eleven approachestraditional as well as innovative. Journal of Documentation, London, v. $58, n$. 4, p. 422-462, 2002.

HJØRLAND, B. What is knowledge organization (KO)?. Knowledge Organization, v. 35, n. 2-3, p. 86-101, 2008.

LEHMKUHL, C. S.; LINDEN, L. L.; BARROS, C. M.; SILVA, E. C. L.; VITAL, L. $P$. Sistemas de organização do conhecimento e Arquivologia: diálogos possíveis. In: BARROS, T. H. B; TOGNOLI, N. B. (orgs). Organização do Conhecimento responsável: promovendo sociedades democráticas e inclusivas: estudos avançados em organização do conhecimento. Belém: Ed UFPA, 2019. p. 58-66

LÓPEZ YEPES, A. Filmotecas y archivos fílmicos en línea: producción, difusión, interconexión y posicionamiento en Internet. Scire, v. 14, n. 2, p. 41-64, 2008.

MAI, J.- E. Analysis in indexing: document and domain centered approaches. Information processing \& management, Elmsford, v. 41. p. 599-611, 2005.

MARCONDES, Carlos Henrique. Uma classificação de tipos de objetos de patrimônio para integração de acervos digitais de arquivos, bibliotecas e museus. Scire. 25:2 (jul.-dic.) 2019.

MARTÍNEZ COMECHE, Juan Antonio. Clasificaciones documentales y procesos informativos: una vía de recionalizaciones. Scire, v. 3, n. 1, p. 31-53, 1997.

MAZZOCCHI, F. Knowledge organization system (KOS). Knowledge Organization, v. 45, n. 1, p. 54-78, 2018.

MCQUEEN, Kelli. Ethical Issues of Knowledge Organization in Designing a Metadata Schema for the Leo Kottke Archives. KO KNOWLEDGE ORGANIZATION, v. 42, n. 5, p. 332-338, 2015.

MILANI, S. O.; PINHO, F. A. Knowledge Representation and Orthophemism: a Reflection Aiming to a Concept. Knowledge Organization, v. 39, p. 384-393, 2012. 
MORALES, F. C.; GARCÍA LÓPEZ F.; GARCÍA, A. M. M.; OHLENSCHLÄGER, K. O. Archivo de Espacio P: propuesta de un cuadro de clasificación para su continuidad digital. Scire, v. 21, n. 2, 2015.

MORENO, A. V. Sistema de información histórica para la colección de papeles varios del Archivo Histórico de la Universidad de Salamanca. Scire, v. 7, n. 2, p. 131-150, 2001.

MORENO, A. V.; MACÍAS, C. S.; GARCÍA, A. M.; CASTRO, A. I. A.; LAIRADO, M. R. La visión de los archivos en el Antiguo Régimen hispano a través de las memorias y relatos de viajeros: análisis de su representación y sistematización metodológica. Scire, v. 11, n. 1, p. 47-64, 2005.

MUNDET, J. R. C.. Los archivos electrónicos en España: del gran hermano al control demográfico. Scire, v. 3, n. 1, p. 75-86, 1997.

PRADO MARTÍNEZ, M. Á. Correspondencias entre la norma aragonesa para la descripción de autoridades de archivos (ARANOR) y MARC21: hacia la web semántica. Scire, 25:1 (en.-jun.) 13-24, 2019.

PRADO MARTíNEZ, M. Á. La Archivística en el ámbito de las organizaciones empresariales: estado de la cuestión y propuestas de actuación. Scire, v. 10, n. 1, p. 185-216, 2004.

PRADO MARTÍNEZ, M. Á. Correspondencias entre la norma aragonesa para la descripción de autoridades de archivos (DARANOR) y MARC21. Scire, v. 25, n.1, p. 13-24, 2019.

REGO, L. M.; SILVA, A. P.; MARTínEZ-AVILA, D.; TOGNOLI, N. B. Aspectos éticos na organização do conhecimento na prática profissional arquivística: um estudo dos princípios de ética da AAB, CIA e SAA. Scire, v. 20, n. 2, 2014.

RIBEIRO, $\mathrm{F}$. The use of classification in archives as a means of organization, representation and retrieval of information. KNOWLEDGE ORGANIZATION, v. 41, n. 4, p. 319-326, 2014.

ROSATI, L.; SCHENA, A.; MASSACESI, R. Childhood and Adolescence Between Past and Present: Using Knowledge Organization to Bridge the Different Channels of a Cultural Institution: The Case of the Istituto degli Innocenti, Fiorenze. KNOWLEDGE ORGANIZATION, v. 40, n. 3, p. 197-204, 2014.

RUBIO HERNÁNDEZ, A. Estudios de usuarios en archivos municipales: una aproximación teórico-práctica. Scire, v. 10, n. 1, p. 217-236, 2004.

SALES, Rodrigo. Classificações bibliográficas e classificações arquivísticas: diferenças e semelhanças na organização do conhecimento. Scire, v. 22, n. 1, 2016. 
SILVA, A. M.; RIBEIRO, F.. Documentation/Information and their paradigms: characterization and importance in research, education, and professional practice. KO KNOWLEDGE ORGANIZATION, v. 39, n. 2, p. 111-124, 2012.

SILVA, A. P.; GUIMARÃES, J. A. C.; TOGNOLI, N. B. Ethical values in archival arrangement and description: An analysis of professional codes of ethics. KO KNOWLEDGE ORGANIZATION, v. 42, n. 5, p. 346-352, 2015.

SILVA, L. S; SCHMIDT, C; M. S. O processo de elaboração do arranjo dos arquivos de Machado de Assis e Rui Barbosa: análise crítica e comparativa. Scire. $24: 2$ (jul.-dic.) 35-44, 2018.

SIMÕES, M. G.; FREITAS, M. C. V.; RODRÍGUEZ-BRAVO, B.. Theory of classification and classification in libraries and archives: convergences and divergences. KO KNOWLEDGE ORGANIZATION, v. 43, n. 7, p. 530-538, 2016.

SMIRAGLIA, R. P. Domain analysis for knowledge organization: tools for ontology extraction. Waltham: Chandos Publishing, 2015.

SMIRAGLIA, R. P. ISKO 11's Diverse Bookshelf: an editorial. Knowledge Organization, v. 38, n.3, p. 179-186, 2011.

SOUZA, R. M. The Representation of Archival Information in Controlled Vocabularies: The Context of the Archival Institutions in Rio de Janeiro. Knowledge Organization, v. 46, n. 7, 2019.

SOUZA, R. M. The Representation of Archival Information in Controlled Vocabularies: The Context of the Archival Institutions in Rio de Janeiro. Knowledge Organization, v. 46, n. 7, p.548-557. 2019.

TOGNOLI N. B.; GUIMARÃES J. A. C.; TENNIS J. T. Diplomatics as a methodological perspective for archival knowledge organization. In: Transition Cultures, Transition KO: Evolving Exploration, Critical Reflection, and Practical Work, NASKO, Milwalkee, v. 1, p. 216-227, 2013.

TOGNOLI, N. B.; RODRIGUES, A. C.; GUIMARÃES, J. A. C. Definindo o conhecimento arquivístico: estruturas conceituais. Informação \& Informação, [S.I.], v. 24, n. 2, p. 58-75, nov. 2019.

TOGNOLI, N. B; BARROS, T. H. B. Os processos de representação do conhecimento arquivístico: elementos históricos e conceituais da classificação e descrição. In: GUIMARÃES, J. A. C.; DODEBEI, V. (Orgs). Organização do conhecimento e diversidade cultural. Marília: ISKO Brasil; FUNDEPE, p. 94-99, 2015.

TOGNOLI, Natalia Bolfarini. A organização do conhecimento arquivístico a partir da Diplomática: elementos históricos e conceituais de um método em revisão. Scire, v. 21, n. 1, 2015. 
TOGNOLI, Natália Bolfarini. Metateoria e Diplomática: a construção de um novo modelo de análise para a identificação de documentos de arquivo. Scire, v. 21, n. 2, 2015.

TROITIÑO RODRÍGUEZ, S. Da crítica diplomática à análise tipológica: abordagens e técnicas de análise documental. Scire, p. 65-72, 2011.

WALT, Van; MARTHINUS, S. A classification scheme for the organization of electronic documents in small, medium and micro enterprises (SMMEs). KO KNOWLEDGE ORGANIZATION, v. 31, n. 1, p. 26-38, 2004.

ZENG, M. L. Interoperability. Knowledge Organization, v. 46, n. 2, p. 122-146, 2019.

ZHANG, Jane. Archival context, digital content, and the ethics of digital archival representation. KO KNOWLEDGE ORGANIZATION, v. 39, n. 5, p. 332-339, 2012.

\title{
KNOWLEDGE ORGANIZATION AND ARCHIVAL SCIENCE: A DOMAIN ANALYSIS IN KNOWLEDGE ORGANIZATION E SCIRE JOURNALS
}

\begin{abstract}
Introduction: Based on a domain analysis, the present article aims to initiate a characterization of the state of the art of Archival Science studies in Knowledge Organization (KO) field. Objectives: To identify the main archival subjects in the epistemological, applied and social perspectives within two important journals: Knowledge Organization and Scire: organización y representacion del conocimiento. To identify the authors and their theoretical influences. Methodology: Exploratory and bibliographical. Domain analysis combining three approaches: epistemological, historical and bibliometric. Results: It shows a balance between epistemological and applied studies and a lack of studies within cultural and social aspects. Regarding the subjects, there is a predominance of studies addressing information technology in the organization and retrieval of digital records, with emphasis on classification studies. Bibliometric analysis identified Duranti and Hjørland as the most influential authors in the publications, confirming their importance in discussions about Archival Science and Knowledge Organization. Conclusion: Finally, it was identified an expressive presence of an Ibero-American discursive community in the studies that aim to approach both areas, evidencing the Spanish, Portuguese and Brazilian pioneering in the admission of archival studies in the scope Knowledge Organization.
\end{abstract}

Descriptors: Knowledge Organization. Archival Science. Domain Analysis. Knowledge Organization Journal. Scire. 


\title{
ORGANIZACIÓN DEL CONOCIMIENTO Y ARCHIVÍSTICA: UN ANÁLISIS DEL DOMINIO EN LAS REVISTAS KNOWLEDGE ORGANIZATION E SCIRE JOURNALS
}

\begin{abstract}
RESUMEN
Introducción: A partir de un análisis de dominio, este artículo busca empezar la caracterización de los estudios archivísticos en la Organización del Conocimiento (OC). Objetivo: Identificar los temas archivísticos en las perspectivas epistemológica, aplicada y cultural en dos importantes vehículos de difusión científica: las revistas Knowledge Organization y Scire: organización y representación del conocimiento. Identificar los autores que producen en los dominios circunscritos y sus influencias teóricas. Metodología: Investigación exploratoria y bibliográfica de naturaleza cualitativa y cuantitativa. Es un análisis de dominio que combina enfoques epistemológicos, históricos y bibliométricos (análisis de citas). Resultados: Demuestran un equilibrio de estudios epistemológicos y aplicados, y una falta de estudios relacionados con aspectos culturales y sociales. En cuanto a los temas, predominan los estudios que abordan las tecnologías de la información en la organización y recuperación de documentos digitales, con énfasis en los estudios sobre clasificación. El análisis bibliométrico identificó a Duranti y Hjørland como los autores más influyentes en las publicaciones, confirmando su importancia en las discusiones sobre Archivística y Organización del Conocimiento. Conclusiones: Se identificó una presencia expresiva de una comunidad discursiva iberoamericana en las discusiones que buscan acercar ambas áreas, destacando el pionero español, portugués y brasileño en la inserción de estudios de archivo en el contexto de la organización del conocimiento.
\end{abstract}

Descriptores: Organización del conocimiento. Archivística. Análisis de domínio. Knowledge Organization Journal. Scire. 